\title{
Predictions of Index of Hemolysis in Shear Blood Flow* (Improvement of Accuracy for Prediction by Modifying Turbulence Model for Orifice-pipe Flow)
}

\author{
Masaaki TAMAGAWA** and Sigeharu MINAKAWA**
}

This paper describes the improvement of accuracy for prediction of index of hemolysis in shear blood flow by computational fluid dynamics (CFD) with modified turbulence model, applying to orifice-pipe blood flow, which is simple model of turbulent shear stress induced hemolysis in the high speed rotary blood pump. Using CFD with partially patched modified $k-\varepsilon$ model (Launder-Kato partially patched model ; LK-Zonal), results of the flow field in the orifice-pipe flow are compared with standard low Reynolds number $k^{-} \varepsilon$ model at whole region (STD), and it is found that the shear stress by LK-Zonal model is predicted more precisely than that by STD model near the impinging flow region. As for hemolysis, from the computational data of orifice-pipe flow, the index of hemolysis is estimated in the same way as our previous paper ${ }^{(1)}$, and it is found that the accuracy for prediction of index of hemolysis is also improved using LK-Zonal model in comparison with STD model, particularly in case of the orificepipe flow with large and long contracted part. From these results, the design method using CFD for the rotary blood pumps, that have impinging flow region to the wall, for suppressing the hemolysis can be proposed.

Key Words: Bio-fluid Mechanics, Turbulent Flow, Shear Stress, Blood Pump, Artificial Heart, Hemolysis, $k-\varepsilon$ Model, Launder-Kato Model

\section{Introduction}

Recently artificial organs, especially rotary blood pumps, have been developed in the worldwide ${ }^{(2)}$, but in this development, hemolysis (red blood cell damage by shear stress) and thrombus occur in the pumps. To suppress the hemolysis and avoid the thrombus is very important and serious problem in developing the rotary blood pumps. In case of developing the artificial heart valve, there are also same problems as hemolysis.

Concerning about the hemolysis, it is considered to be caused by shear stress and exposure time near the device, like high-speed rotational blade of pumps, in the internal flow ${ }^{(3)-(6)}$. Hellums et al. ${ }^{(3)}$ arranged the

* Received 19th June, 2000

** Department of Energy Conversion Science, Graduate School of Energy Science, Kyoto University, Sakyoku, Kyoto606-8501, Japan. E-mail : tama@energy. kyoto-u.ac.jp previous researcher's literature and showed that the threshold of hemolysis is dependent on both shear stresses and exposure time (Fig. 1). There are two kinds of shear stress in high shear flow like the flow in



Fig. 1 Concept of hemolysis with shear stress and exposure time (Hellums et al.) 




(a) Laminar shear flow

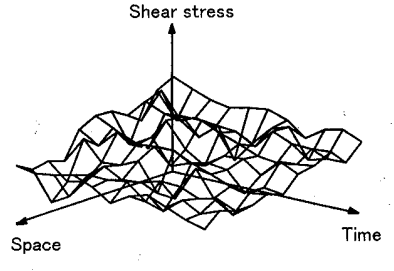

(b) Turbulent shear flow
Fig. 2 Concept of laminar and turbulent shear stress in time and space

the rotary blood pump. One is molecular viscous shear stress and the other is turbulent shear stress (shear component of Reynolds stress). In the high shear flow, the turbulent shear stress cannot be ignored because it is much larger than molecular shear stress. There are some papers describing predictions of hemolysis ${ }^{(7),(8)}$ using the shear stress including turbulent shear stress, exposure time and fatigue factor etc., but the agreements between the predictions and the experiments have not been complete yet. Additionally, the flow fields in the pumps are complicated as shown in Fig. 2, this is also a kind of reasons why the agreements are not complete.

By the way, computational fluid dynamics (CFD) has been widely used to assist design and developing the fluid machine, such as rotary pumps. Most cases of driving conditions in the pumps, there are high shear and turbulence in the flow, and it is necessary to analyze internal flow without computational cost. In the same way, the design using CFD is also important tool for developing blood pumps. Therefore, it is more necessary to develop CFD code for design and predicting hemolysis properties without large cost. In our previous paper ${ }^{(1)}$, the prediction has been tried using CFD with standard turbulence model, but the prediction has not been completed even if the simple orifice-pipe flow is dealt. The reasons why it was not complete are considered to be that there are some effects, they are (1) anisotropy turbulence in the separated flow, (2) high concentrated suspension, and ( 3 ) direct contact between the red blood cell and the wall. As for (1) anisotropy of turbulence, the turbulence model itself has many problems to express this effect in this study. Concerning about (2) the fluid is high concentrated suspended flow (the Hematocrit number, that is volumetric ratio of red blood cells in blood, is $40 \%$ ), interactions among the red blood cells are large, and it is too complicated the phenomena itself. It is not easy to construct the model for this suspension. Additionally, it is also difficult to get proper model coefficient from the experiments. Therefore, the fluid is regarded as low concentrated and isotropic turbulent flow in this study. As for ( 3 ) the effects of the wall-contact and particle inertia, our previous paper ${ }^{(8)}$ shows that they are large factor for hemolysis predictions, but it was found that it is necessary to overestimate the particle size to predict the hemolysis precisely. So it is more necessary to improve accuracy of index of hemolysis without considering ( 1 ), (2) and ( 3 ).

Back to the origin, in this paper, the modifications of the turbulence model are focused because the orifice flow has separation, reattached, and impinging flow to wall. Especially for impinging flow to internal wall, the generation term of transport equations for turbulence energy $(k)$ is overestimated at the impinging region by the standard $k-\varepsilon$ model, and the quantity of turbulent energy and turbulent shear stress are also oyerestimated in comparison with experiment, and this is a kind of defects of standard $k-\varepsilon$ model. Therefore, it is necessary to modify the model for overcoming the defect without large computational cost.

Our main goal is to predict hemolysis by turbulent shear stress occurs in the rotary blood pumps. In this paper, to improve the accuracy of prediction of hemolysis, the flow fields are computed using modified $k-\varepsilon$ model (Launder-Kato partially patched model), which is more adequate for the impinging flow to the wall than that using the well-known standard $k-\varepsilon$ model, and it's accuracy is discussed. As for the objective configurations, to compare with the effects of the previous discussed factors, the orifice-pipe flow is used that had already been examined as the model for the flow near the impeller in the rotary blood pump. It is expected to get the fundamental understandings about the effects of the flow fields on accuracy of the prediction of hemolysis and method for CFD design to develop the blood pumps.

\section{Objective Configurations for Analysis and Hemolysis Tests}

In this study, three kinds of configurations (A, B and $\mathrm{C}$ ) for the orifice are used and two directions ( $\mathrm{F}$ : Forward and B: Backward), in this paper especially configurations $\mathrm{AB}, \mathrm{AF}, \mathrm{BB}, \mathrm{BF}, \mathrm{CB}$ is used for hemolysis test (Fig. 3 (a)). These configurations can be used for the experiments to explain the effects of round for edge on the wall or the front and back of the orifice on the hemolysis. Figure $3(\mathrm{~b})$ shows the hemolysis circuits that consists of blood pump (Biopump, made by Bio-Medico Co. Ltd.), reservoir bag for the blood and the orifice pipe. The flow rate for the pump is $5 \mathrm{l} / \mathrm{min}$ according to the working conditions for the rotary blood pump. In addition, pressure loss is from 150 to $300 \mathrm{mmHg}$ in every configuration of the orifice, and in order to keep the pressure losses in 

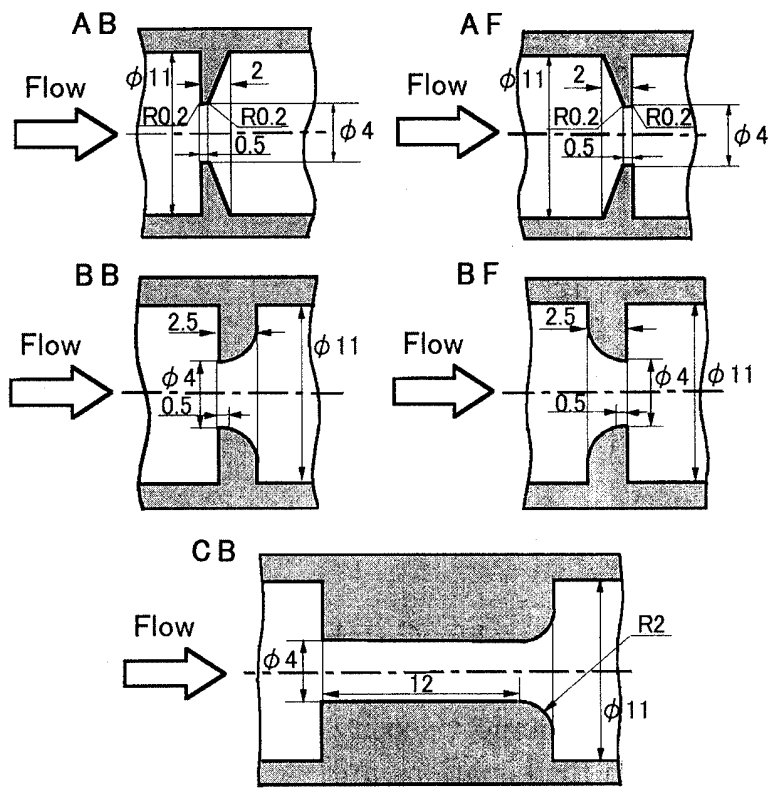

(a) Five configurations of various orifices

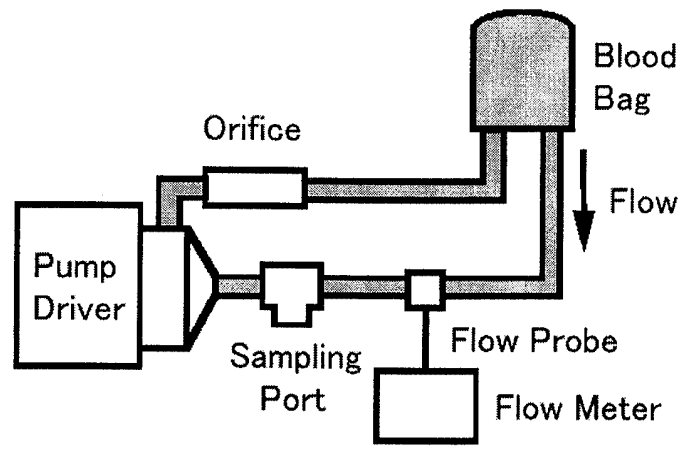

Circuit A (Orifice BB)

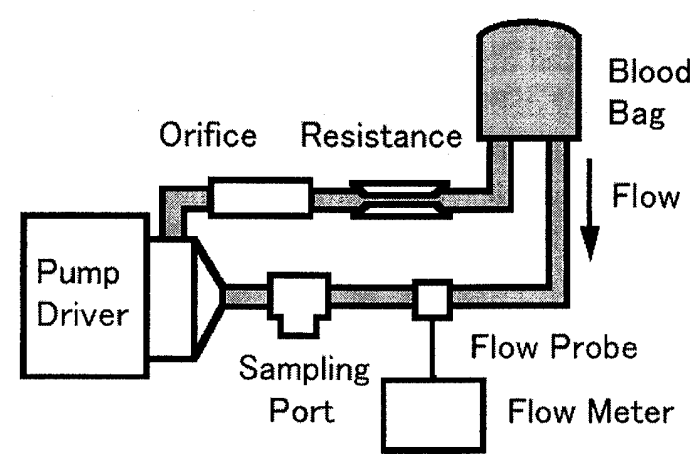

Circuit A (Orifice AB, AF, BF, CB)

(b) Experimental circuits for hemolysis test (Configuration $\mathrm{BB}$ and others)

Fig. 3 Configurations of various orifices and experimental circuits for hemolysis test

every hemolysis circuit the variable resistance whose configuration is small and long round shaped are used. To decrease the effects of the differences for the blood in the each circuit, the two Bio-pumps should be used
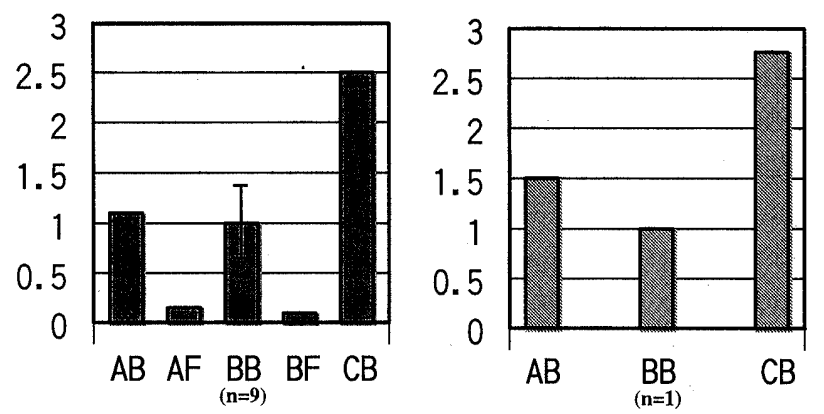

(a) Relative Index of Hemolysis for red blood cells

(b) Relative Index of Hemolysis for microcapsules

Fig. 4 Experimental results for hemolysis tests ( $n$ : number of samples)

in the hemolysis circuit at the same time. In this case, Circuit A (Configuration BB) is dealt as standard circuit for hemolysis and the pressure loss is 300 mmHg. In this experiment, micro capsules (Fuji Film Co Ltd.) including dye stuff, which is a kind of engineering model for cells, are used in the same conditions to compare with red blood cells.

As for the hemolysis test, the modified Durabkin method is used to examine the quantity of free hemoglobin concentration in plasma. In case of microcapsules, the dyestuff from internal cells is tested. In this study, rate of hemolysis (degree of cell damage per unit time) is used to express hemolysis properties, which are defined as follows:

$$
\text { I.H. }=\frac{\left(\mathrm{Hb}\left(T_{1}\right)-\mathrm{Hb}\left(T_{0}\right)\right) \times(100-\mathrm{Ht}) \times V}{Q \times D T}(\mathrm{mg} / \mathrm{dl})
$$

Here I.H. means index of hemolysis, $\mathrm{Hb}(T)(\mathrm{mg} / \mathrm{dl})$ means free hemoglobin concentration at time $T, T_{1}$ $(\min )$ means the time for getting the sample blood from the circuit, $T_{0}(\mathrm{~min})$ means starting time for the hemolysis test, $D T=T_{1}-T_{0}(\min ), V(1)$ means the blood volume to fill the circuit, and $Q(1 / \mathrm{min})$ is flow rate. In this paper, relative index of hemolysis (R.I.H.) using standard circuit (Configuration BB) is defined as follows:

$$
\text { R.I.H. }=\frac{\text { I.H.test }}{\text { I.H.BB }}
$$

The reason why this definition should be used is that if we use this definition the effects of the factor without physical factors are large in this test. Concerning about the microcapsules, I.H. and R.I.H are defined in the same process using other drugs.

Figure $4(\mathrm{a})$ shows the relative indexes of hemolysis for red blood cells at each configuration $(\mathrm{AB}, \mathrm{AF}, \mathrm{BB}, \mathrm{BF}$ and $\mathrm{CB})$ under the condition that flow rate is $5 \mathrm{l} / \mathrm{min}$. Figure 4 (b) shows the relative indexes of hemolysis for micro capsules including internal stuff tested to compare engineering model 
with red blood cells. In each result, it is found that the amount of hemolysis for the configuration $\mathrm{CB}$ is from 2 to 2.5 times as that for the configuration $\mathrm{BB}$, and the amount of $\mathrm{AB}$ is a little bit large in comparison with that for $\mathrm{BB}$. In the contrary, it is found that the hemolysis of $\mathrm{AF}$ and $\mathrm{BF}$ is much less than that for $\mathrm{BB}$, and nearly 0 . The tendency of damage for microcapsule is similar to that of red blood cells from hemolysis test.

\section{Modification of Turbulence Model and Computational Results}

\section{1 Governing equations and turbulence model}

The computational conditions are that flow rate $Q=51 / \mathrm{min}$, inlet flow velocity $U_{\text {in }}=0.877 \mathrm{~m} / \mathrm{s}$ and diameter $D(=11 \mathrm{~mm})$ is present scale. Coordinate system is cylindrical one, and $x$-direction is parallel to the flow and $r$-direction is radial component of the pipe. The diameter of contracted region is $4 \mathrm{~mm}$, and Reynolds number is 10000 based on diameter $D$ and viscosity of water.

Considering that the length scale of a vortex is as same order as diameter of a red blood cell, the conditions for this analytical model are supposed in the following way:

(i ) The number of the red blood cells is small, and there are no aggregations by mutual interactions among particles

(ii) Red blood cells are spherical particle, and they move along streamline.

(iii) The movement of the particles is parallel motion without rotation.

These are same conditions as in our previous papers. Using these assumptions, the turbulence models are shown and discussed.

3.1.1 Standard $k-\varepsilon$ model for low Reynolds number model (STD) In our previous papers, standard low-Reynolds number $k-\varepsilon$ model proposed by Abe-Nagano (STD) has been used as a turbulence model for the computation, that can be used for the separated and reattached flow. The governing equations are continuum equation, momentum equations, and transport equations of $k$ (turbulent energy), $\varepsilon$ (energy dissipation) in cylindrical coordinate system. They are shown as follows ${ }^{(9)}$ :

$$
\begin{aligned}
& \frac{\partial u}{\partial x}+\frac{1}{r} \frac{\partial(r v)}{\partial r}=0 \\
& \frac{\partial u}{\partial t}+u \frac{\partial u}{\partial x}+v \frac{\partial u}{\partial r}=-\frac{\partial p}{\partial x}+\left(\frac{1}{R e}+\nu_{t}\right) \nabla^{2} u \\
& \quad+2 \frac{\partial \nu_{t}}{\partial x} \frac{\partial u}{\partial x}+\frac{\partial \nu_{t}}{\partial r}\left(\frac{\partial u}{\partial r}+\frac{\partial v}{\partial x}\right) \\
& \frac{\partial v}{\partial t}+u \frac{\partial v}{\partial x}+v \frac{\partial v}{\partial r}=-\frac{\partial p}{\partial r}+\left(\frac{1}{R e}+\nu_{1}\right)\left(\nabla^{2} v-\frac{v}{r^{2}}\right)
\end{aligned}
$$

$$
+2 \frac{\partial \nu_{t}}{\partial r} \frac{\partial v}{\partial r}+\frac{\partial \nu_{t}}{\partial x}\left(\frac{\partial u}{\partial r}+\frac{\partial v}{\partial x}\right)
$$

And transport equations are

$$
\begin{aligned}
& \frac{\partial k}{\partial t}+u \frac{\partial k}{\partial x}+v \frac{\partial k}{\partial r}=\frac{1}{R e} \nabla^{2} k \\
& \quad+\frac{1}{\sigma_{k}}\left(\frac{\partial \nu_{t}}{\partial x} \frac{\partial k}{\partial x}+\frac{\partial \nu_{t}}{\partial r} \frac{\partial k}{\partial r}\right) \\
& \frac{\partial \varepsilon}{\partial t}+u \frac{\partial \varepsilon}{\partial x}+v \frac{\partial \varepsilon}{\partial r}=\frac{1}{R e} \nabla^{2} \varepsilon \\
& \quad+\frac{1}{\sigma_{\varepsilon}}\left(\frac{\partial \nu_{t}}{\partial x} \frac{\partial \varepsilon}{\partial x}+\frac{\partial \nu_{t}}{\partial r} \frac{\partial \varepsilon}{\partial r}\right)+C_{\varepsilon 1} \frac{\varepsilon}{k} G-C_{\varepsilon 2} f_{\varepsilon} \frac{\varepsilon^{2}}{k} \\
& \nu_{t}=C_{\mu} f_{\mu} \frac{k^{2}}{\varepsilon} \\
& C_{\mu}=0.09, C_{\varepsilon 1}=1.5, C_{\varepsilon 2}=1.9, \quad \sigma_{k}=1.4, \quad \sigma_{\varepsilon}=1.4
\end{aligned}
$$

where $\nu_{t}$ is dynamic viscosity of turbulence, and the constants in the equations are

$$
\begin{aligned}
& f_{\mu}=\left\{1-\exp \left(-\frac{y^{*}}{14}\right)\right\}\left[1+\left(\frac{5}{R_{t}^{3 / 4}}\right) \exp \left\{-\left(\frac{R_{t}}{200}\right)^{2}\right\}\right] \\
& f_{\varepsilon}=\left\{1-\exp \left(-\frac{y^{*}}{3.1}\right)\right\}\left[1-0.3 \exp \left\{-\left(\frac{R_{t}}{6.5}\right)^{2}\right\}\right] \\
& y^{*}=\frac{u_{\varepsilon}}{\nu}, R_{t}=\frac{k^{2}}{\nu \varepsilon}, u_{\varepsilon}=(\nu \varepsilon)^{1 / 4}
\end{aligned}
$$

$u_{\varepsilon}$ is Kolmogolov's velocity scale. In Eq. ( 7 ) generation term $G$ is expressed as follows :

$$
G=\nu_{t} S^{2}
$$

where $S$ is strain velocity,

$$
S=\sqrt{\left[2\left\{\left(\frac{\partial u}{\partial x}\right)^{2}+\left(\frac{\partial v}{\partial r}\right)^{2}+\left(\frac{v}{r}\right)^{2}\right\}+\left(\frac{\partial u}{\partial r}+\frac{\partial v}{\partial x}\right)^{2}\right]} .
$$

It is generally known that this generation term is overestimated at impinging region to wall using standard $k-\varepsilon$ model. Therefore, in cases of our objects, there are some regions in front of the contracted part.

\subsubsection{Launder-Kato model for impinging flow to} internal wall(LK) To overcome this defect, Launder and Kato proposed the modification model of the generation term in $k$-equation $(\mathrm{LK})^{(10)}$.

$$
G=\nu_{t} S \Omega
$$

where $S$ is strain velocity and $\Omega$ is vorticity as follows :

$$
\Omega=\sqrt{\left(\frac{\partial u}{\partial r}-\frac{\partial v}{\partial x}\right)^{2}}
$$

This model is suitable for external flow including imping flow region, such as the flow around a buliding. But according to the following section, it is not suitable in case of high shear and large generation, such as large contraction ratio orifice-pipe flow.

3.1.3 Launder-Kato partially patched model (LK-Zonal) In this study, the Launder-Kato partially patched model ( $\mathrm{LK}-$ Zonal) is proposed. That is 
to apply LK model for the impinging flow region, and to apply STD model for whole region except impinging flow region as follows:

$$
G= \begin{cases}\nu_{t} S^{2} & \text { (STD Model for whole region) } \\ \nu_{t} S \Omega & \text { (LK Model for impinging flow region) }\end{cases}
$$

For example, Fig. 5 shows the typical zone for modifying turbulence model in the configuration $\mathrm{AB}$. In this figure, we adapt the LK model to the orifice front, and STD model to others. To determine the boundary of the zone, the ratio between vorticity $\Omega$ and strain velocity $S$ should be checked to prevent perturbations of the flow driven by large vorticity outside the LKzone. Figure 6 shows contour line of strain velocity in each model (STD model, LK model, LK-Zonal model) for configuration $\mathrm{AB}$. In this figure shading zone means that the contour is larger than 200. From this figure, it is found that the small turbulence of strain velocity occurs from the edge of orifice to the downstream. From the point of view of computational

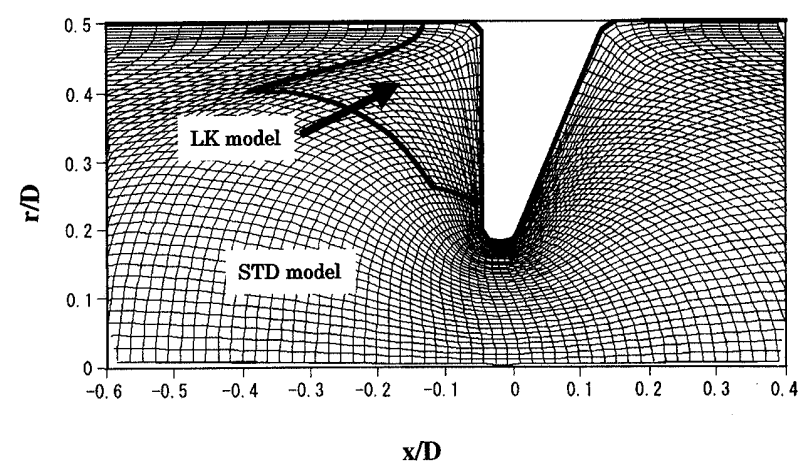

Fig. 5 Typical zone for modifying turbulence model in the configuration $\mathrm{AB}$

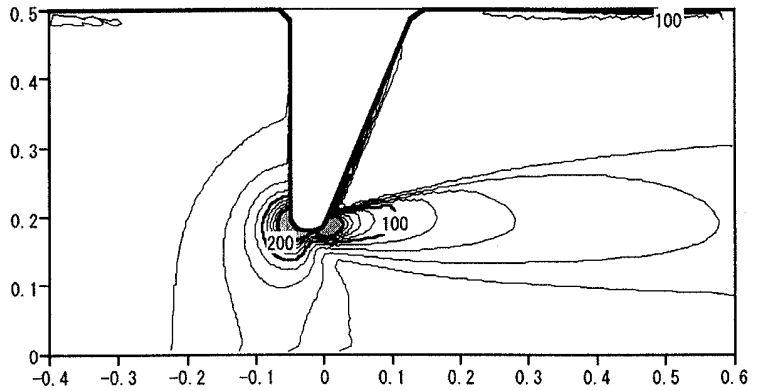

(a) STD model

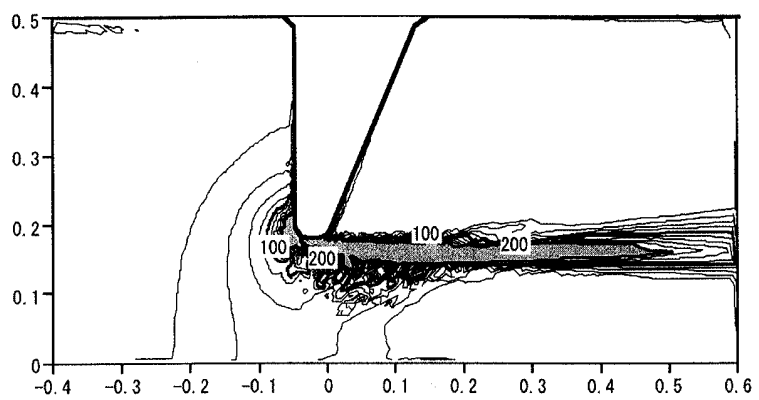

(b) LK model

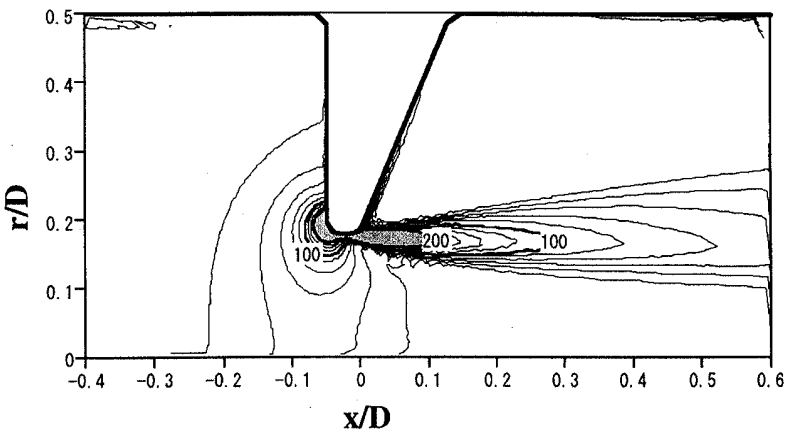

(c) LK-Zonal model

Fig. 6 Contour line of strain velocity in case of $A B$ and effects of zoning

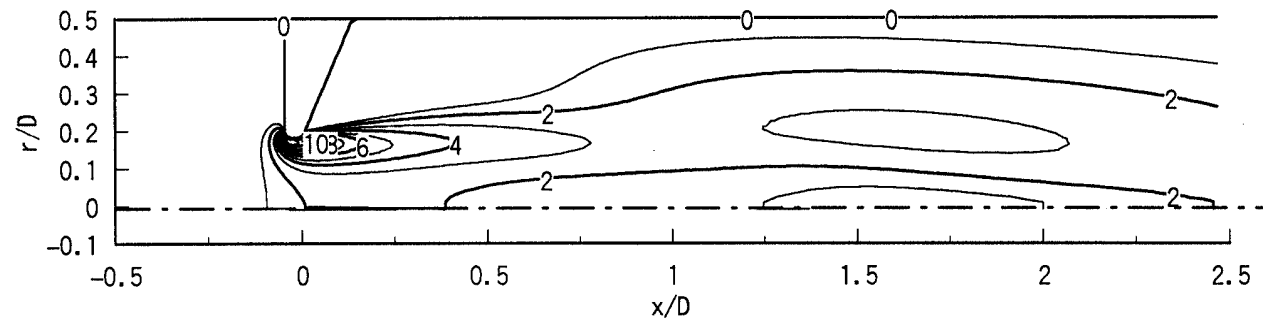

(a) STD model

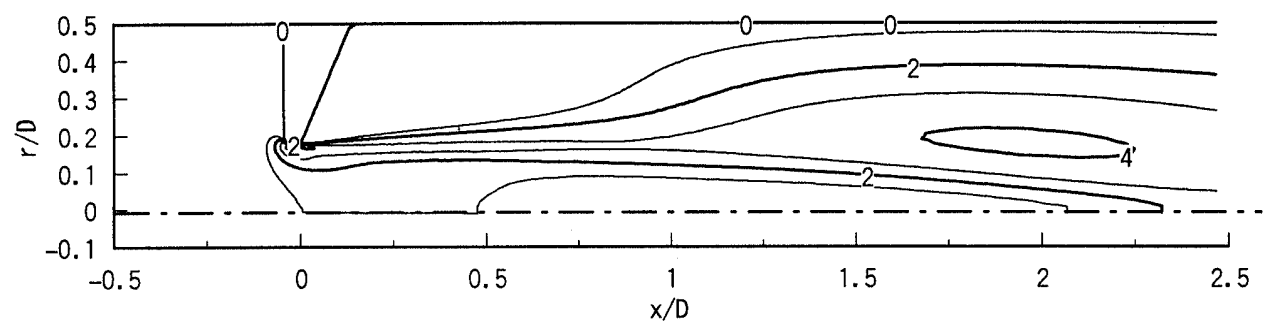

(b) LK-Zonal model

Fig. 7 Contour line of turbulence energy in case of $A B$ 


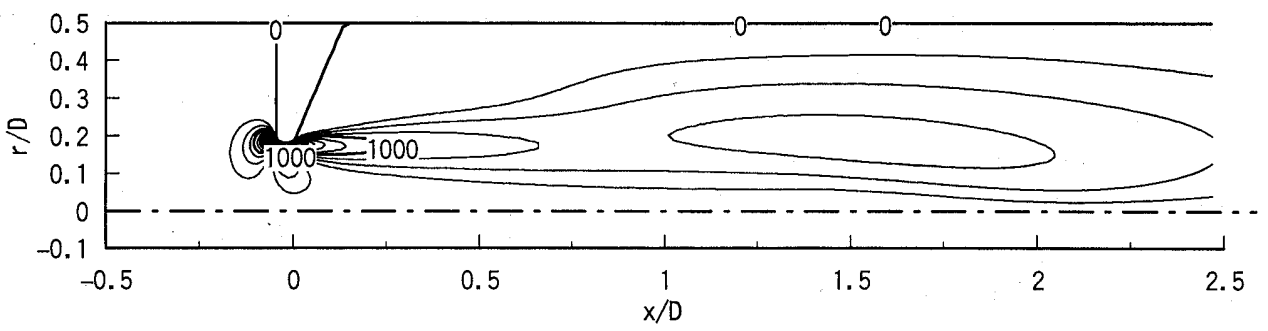

(a) STD model

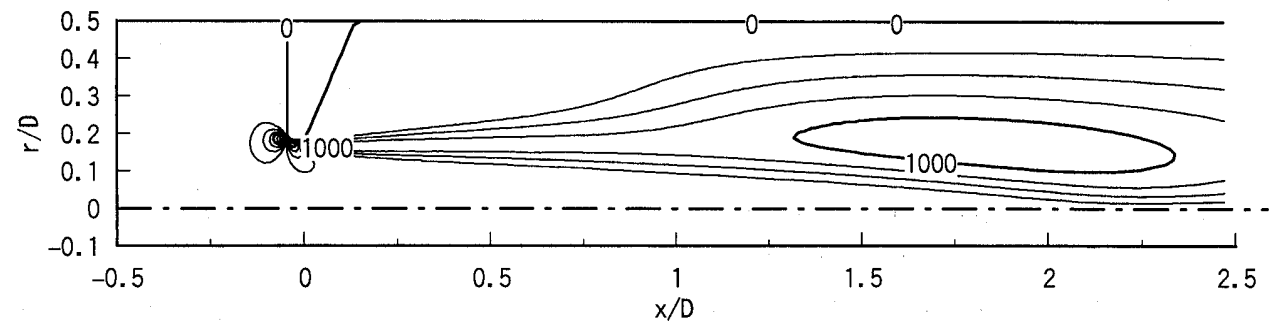

(b) LK-Zonal model

Fig. 8 Contour line of turbulent shear stress in case of $\mathrm{AB}$

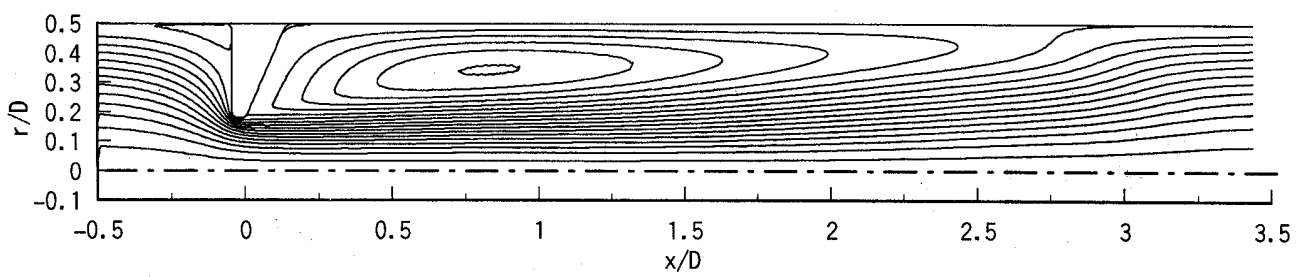

(a) STD model

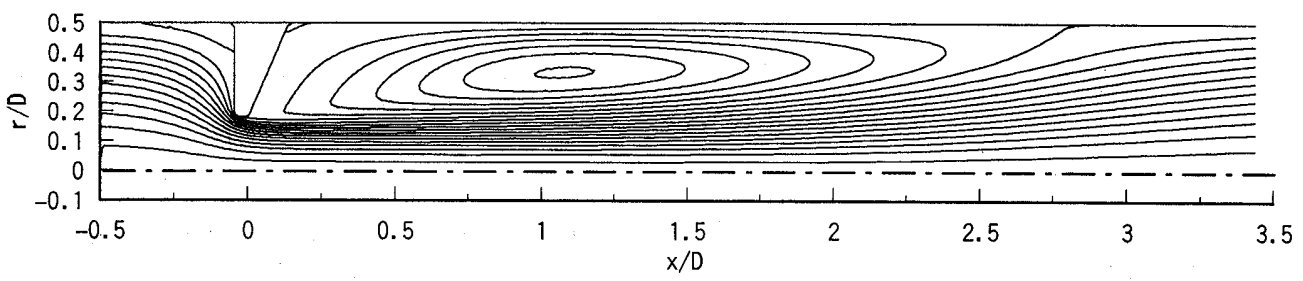

(b) LK-Zonal model

Fig. 9 Streamline in case of $\mathrm{AB}$

accuracy, it is not suitable to use LK model to the whole region from this result. From the following section, STD and LK-Zonal models are compared and discussed, especially for configuration $\mathrm{AB}$.

\section{2 Computational results}

Figure 7 shows the contour line of turbulent energy in case of configuration AB. From this figure, it is found that peak turbulent energy at the edge of the orifice is decreasing in LK-Zonal model compared with that in STD model. This decreasing is owing to modifying the generation term in $k$-equation. And it is found that the second peak in the downstream is increasing in LK-Zonal model. This makes effects on the predicted index of hemolysis, which is discussed later. Figure 8 shows the contour line of turbulent shear stress in case of configuration $\mathrm{AB}$. In the same way as turbulent energy, peak turbulent shear stress is decreasing from 5900 to $1840 \mathrm{~Pa}$ using LK-Zonal model. Figure 9 shows streamline in case of configuration $\mathrm{AB}$. In this figure, it is found that the separation point at the front edge of the orifice in $\mathrm{LK}^{-}$ Zonal model moves to upstream a little bit, and the pattern of streamline near the reattachment point and the center of re-circulating vortex is different between STD and LK-Zonal model

\section{Discussions about Hemolysis}

\section{1 Method for predicting hemolysis}

In this section, the predictions for hemolysis are done using numerical data obtained from the computational results. In our method, the flow is steady and the red blood cells move along the stream line, then it is not necessary to deal cell damage by Lagrangian method, and Eulerian method are used. 
Considering that the damage of red blood cells can be estimated using the integration of the shear stress (turbulent shear stress), that is more than proper threshold level, multiplied by flow rate with path line, the estimation quantity $P\left(\tau_{\mathrm{th}}\right)$ (predicted index of hemolysis) that has been developed ${ }^{(1),(8)}$ is shown as follows :

$$
\begin{aligned}
& P\left(\tau_{\mathrm{th}}\right)=\iint \tau \Delta L \Delta Q=\iint \tau U \frac{\Delta S}{\Delta Q} \Delta Q=\iint \tau U \Delta S \\
& \tau\left(\tau_{t}, \tau_{\mathrm{th}}\right)=\left\{\begin{array}{cc}
0 & \left(0 \leq\left|\tau_{t}\right|<\tau_{\mathrm{th}}\right) \\
\left|\tau_{t}\right| & \left(\left|\tau_{t}\right|>\tau_{\mathrm{th}}\right)
\end{array}\right.
\end{aligned}
$$

Here $\tau_{t}$ is turbulent shear stress, $\tau_{\mathrm{th}}$ is threshold shear stress, $U, \Delta L, \Delta Q, \Delta S$ are velocity, micro length, micro flow rate and micro area as shown in Fig. 10. It should be noticed that the $P$ is not index of hemolysis (I.H.) itself like Eq. ( 1 ), but it is proportional to I.H.

\section{2 Results for prediction compared with experi- ments}

Using above method, the predicted index of hemolysis $P$ (predicted I.H.) of each configuration is estimated. Figure 11 shows the predicted I.H. with

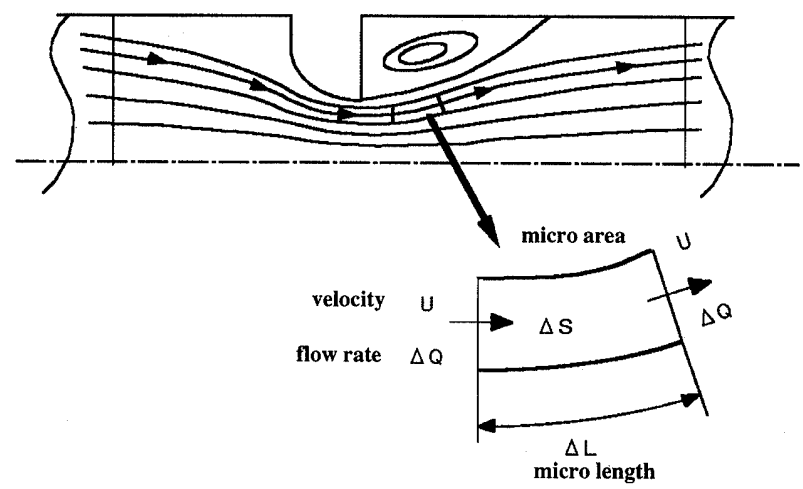

Fig. 10 Typical concept of hemolysis with streamline

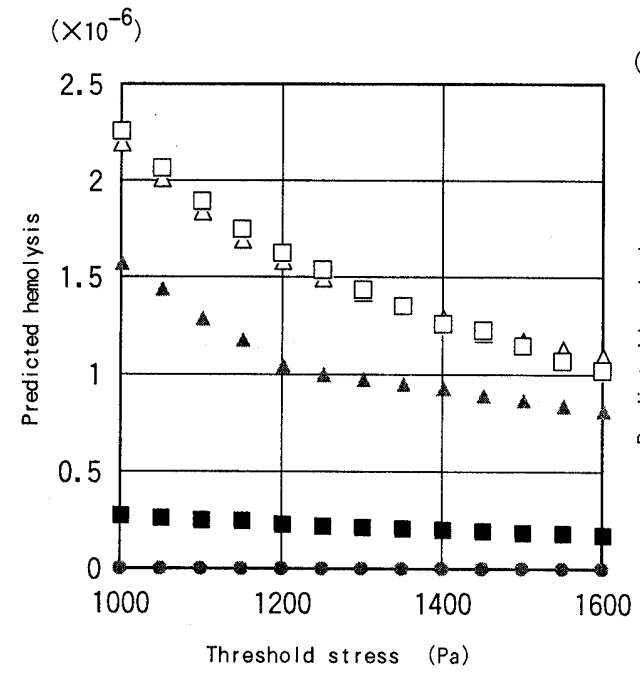

(a) STD model

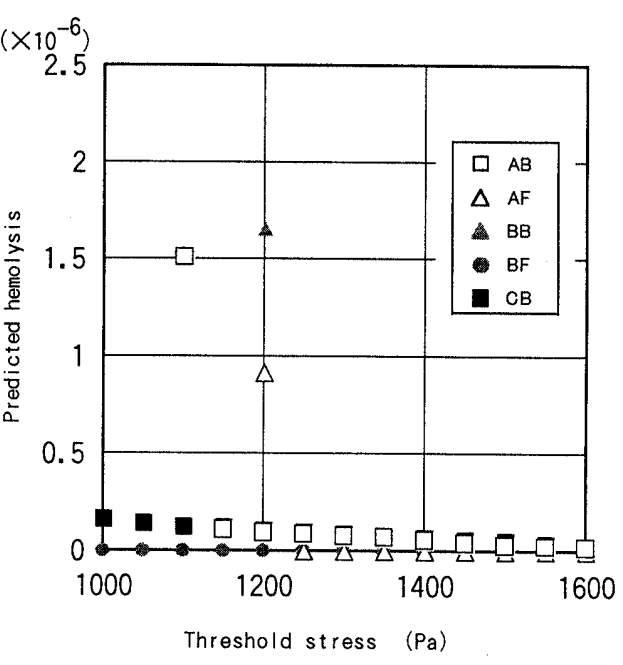

(b) LK-Zonal model

Fig. 11 Predicted Index of Hemolysis (I.H.) with threshold stress $\tau_{\mathrm{th}}$

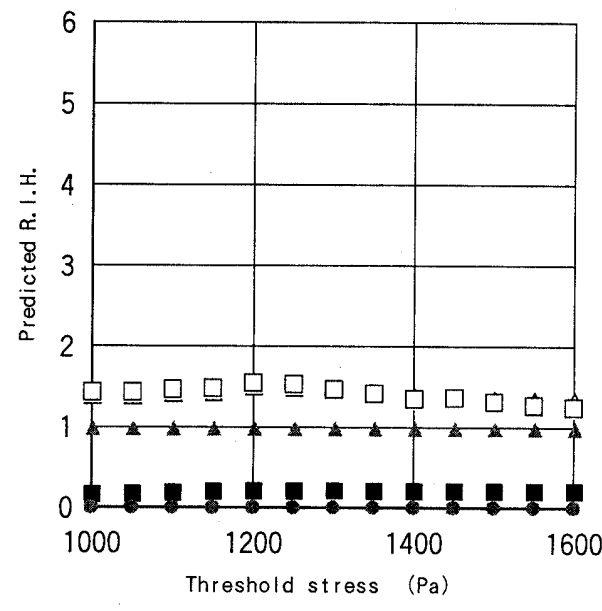

(a) STD model

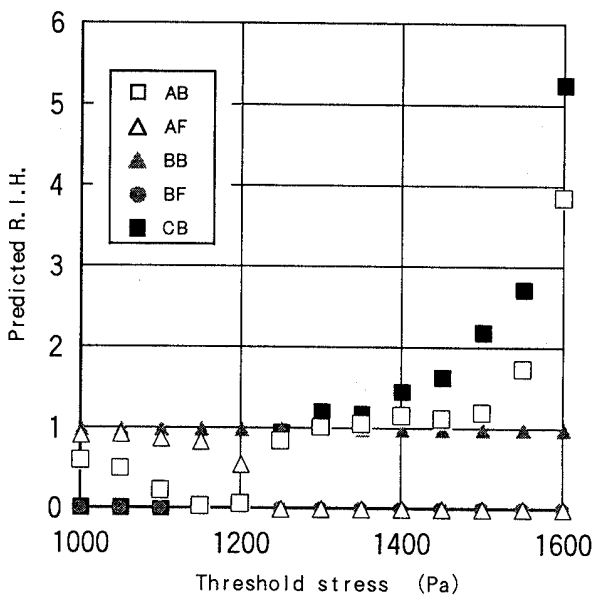

(b) LK-Zonal model

Fig. 12 Predicted Relative Index of Hemolysis (R.I.H.) with threshold stress $\tau_{\text {th }}$ 


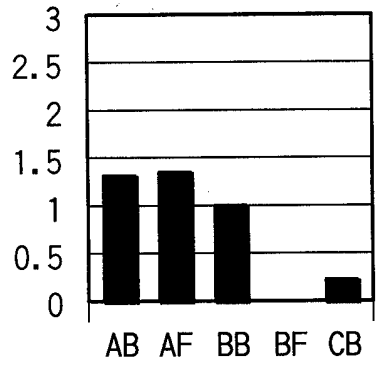

(a) STD model

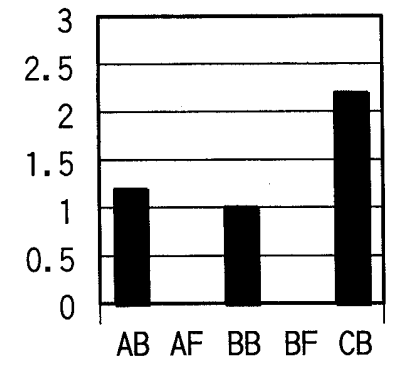

(b) LK-Zonal model
Fig. 13 Comparison between STD model-predictions and LK-Zonal model-predictions using threshold turbulent shear stress $\left(\tau_{\mathrm{th}}=1500 \mathrm{~Pa}\right)$

threshold stress $\tau_{\text {th }}$. It is found that the predicted I.H. for $\mathrm{AB}, \mathrm{AF}, \mathrm{BB}$ in $\mathrm{LK}-$ Zonal model are suddenly changing from the threshold stress 1000 to $1200 \mathrm{~Pa}$, and near to 0 at more than $1200 \mathrm{~Pa}$. Compared with LK-Zonal model, the predicted I.H. in STD model for the same configurations are decreasing slowly with threshold stress. Figure 12 shows predicted relative index of hemolysis (predicted R.I.H.) with threshold stress $\tau_{\text {th. }}$ In contrast, predicted R.I.H. for all configurations in STD model is not so changed even if changing the threshold stress. On the other hand, predicted R.I.H in LK-Zonal model is changing variously with threshold stress. When the threshold stress is more than $1200 \mathrm{~Pa}$, predicted R.I.H for $\mathrm{AF}$ and $\mathrm{BF}$ are decreasing because the turbulent shear stress is decreasing at impinging flow region using $\mathrm{LK}-$ Zonal model.

Changing the threshold stress and comparing with the experimental results in hemolysis tests, it is found that the threshold of shear stress $\tau_{\text {th }}$ is nearly $1500 \mathrm{~Pa}$ for optimizing the predicted R.I.H in both models. According to this optimization, the predicted relative indexes of hemolysis in each configuration are shown in Fig. 13. It is found that using LK-Zonal model the accuracy of predicted R.I.H is improving for especially configuration $\mathrm{CB}$ and $\mathrm{AF}$ in comparison with that in STD model. The reason why the accuracy of predicted R.I.H is improving is owing to suppressing the overestimated turbulent shear stress at impinging flow region. From these results, it is shown that using LK-Zonal model, which is partially patched LK model, the accuracy become improving. In the next step, the ratio between the effects of the turbulence model of the flow and the cell's contact with wall should be studied.

\section{Conclusions}

In this study, in order to simplify the hemolysis phenomena in the rotary blood pump, the special turbulent shear flows using the orifice-pipe are prepared. (i) The red blood cells are broken by shear stress (turbulent shear stress: Reynolds shear stress), (ii) the hemolysis occurs at this orifice-pipe flow compared with other part in the hemolysis circuit, (iii) the index of hemolysis can be estimated using the integration of the shear stress, that is more than proper threshold level, multiplied by flow rate with path line, were supposed. Under these conditions, the following things were concluded in this paper:

(1) Accuracy of predicted index of hemolysis with LK-Zonal model was improved better than that with previous standard low-Reynolds number $k-\varepsilon$ model, in case of analysis of the flow including impinging flow region. The agreement between prediction and experiment (hemolysis tests) was good.

(2) To apply this method to design the pumps, it was recommended that after (i) computing the whole flow region with standard low-Reynolds number $k-\varepsilon$ model and finding the impinging flow region, and (ii) patching LK-model to the impinging flow region and re-computing, (iii) index of hemolysis was predicted using our proposed estimation.

In future, this method will be applied to the computation in the configuration of the actual rotary blood pumps (artificial organs), and then these results with the actual hemolysis test in developing the artificial organs will be compared.

\section{Acknowledgments}

A part of this study was supported by a Grantin-Aid for Scientific Research from the Ministry of Education, Science, Sports and Culture, Japan.

\section{References}

(1) Tamagawa, M., Saitoh, K. and Akamatsu, T., Predictions of Hemolysis in Turbulent Shear Orifice Flow, Artif Organs, Vol. 20 (1996), pp. 553559.

(2) Akamatsu, T., Tsukiya, T., Nishimura, K., Park, C.H. and Nakazeki, T., Recent Studies of the Centrifugal Blood Pump with a Magnetically Suspended Impeller, Artif Organs, Vol. 19 (1995), pp. 631-634.

( 3 ) Hellums, J.R., Cardiovascular Flow Dynamics and Measurements, (1977), University Park Press.

(4) Croce, P.A., Hemolysis of Erythrocyte in Laminar and Turbulent Shear Flows, Ph.D. Thesis, (1972), Washington University.

(5) Hashimoto, S., Erythrocyte Destruction under Periodically Fluctuating Shear Rate: Comparative Study with Constant Shear Rate, Artif Organs, Vol. 13 (1989), pp. 458-463.

(6) Affeld, K., Reininger, A.J., Gadischke, J., Grunert, K., Scmidt, S. and Thiele, F., Fluid Mechanics of the Stagnation Point Flow Chamber and Its Platelet Deposition, Artif Organs, Vol. 19 (1995), pp. 597-602. 
(7) Umezu, M., Design Optimization of Artificial Heart Blood Pumps Based on Hemolysis Testing, ASME Adances in Bioengineering, Vol. 28, BED (1994), p. 33.

(8) Tamagawa, M., Akamatsu, T. and Sano, T., Prediction of Hemolysis in Turbulent Shear Orifice Flow, JSME Int. J. Ser. B, Vol. 43, No. 2 (2000), pp. 225-232.

(9) Abe, Y. and Nagano, T., An Improved $k^{-} \varepsilon$ Model for Prediction of Turbulent Flows with Separation and Reattachment, Trans. Jpn. Soc. Mech. Eng., (in Japanese), Vol. 58. No. 4, (1992), pp. 30033010.

(10) Kato, M. and Launder, B.E., The Modeling of Turbulent Flow around Stationary and Vibrating Square Cylinders, Proc. of 9th Symp. Turbulent Shear Flows, (1993), pp. 1041-1046. 